patients. This can be done by integrating clinical phenotype with genetic setup, gene expression profile and analysis of activated pro inflammatory pathways.

\section{CLINICAL TRIALS WITH IFN BLOCKERS}

Richard Furie. Division of Rheumatology, Northwell Health, Great Neck, USA

\subsection{6/lupus-2020-eurolupus.9}

Background Type I interferon (IFN) pathway activation has long been demonstrated in patients with systemic lupus erythematosus (SLE). The target of drug development in SLE, approaches to inhibit type I IFN have been quite eclectic. The initial strategy, which utilized a monoclonal antibody to interferon-alpha, was not successful as the phase 2 study in SLE with rontalizumab failed to achieve the primary end point of the study. Shortly thereafter, the results of a phase 2 SLE study with sifalimumab, a second monoclonal antibody to interferon-alpha, were released. While benefit was achieved, the pharmacodynamic and clinical effects were not as robust as those attained in a phase 2 SLE study with anifrolumab, an antibody to the type I IFN receptor that inhibits all type I IFNs. The phase 3 anifrolumab program was comprised of two studies, known as TULIP 1 and TULIP 2. TULIP 1 evaluated two doses (150 and $300 \mathrm{mg}$ ) administered intravenously every 4 weeks through week 48 with the primary end point, SLE Responder Index response rate, at week 52. Although this study failed to achieve the primary end point, it was recognized after unblinding that $8 \%$ of study subjects were misclassified as non-responders because of NSAID use. While post-hoc revisions to the restricted medication rules did not change the primary outcome of TULIP 1, these modifications did result in several successful secondary outcomes, including the British Isles Lupus Assessment Group-based Composite Lupus Assessment response rates (BICLA: placebo [29.6\%] vs anifrolumab $300 \mathrm{mg}$ [46.1\%]). An additional outcome of the post-hoc evaluation of TULIP 1 was the modification to the TULIP 2 primary end point.

TULIP 2's initial design was identical to TULIP 1 with the exception that just one dose of anifrolumab (300 $\mathrm{mg}$ ) was compared to placebo. However, before unblinding, the end point was switched from SRI at week 52 to BICLA response rate at week 52. TULIP 2 not only achieved the primary outcome (BICLA: placebo [31.5\%] vs anifrolumab $300 \mathrm{mg}$ [47.8\%]), but multiple secondary end points were also attained, chief of which were the ability to taper corticosteroids as well as improvement of cutaneous disease activity. Safety signals of note included a higher rate of herpes zoster reactivation (placebo: 1.1\% vs anifrolumab: 7.2\%).

While not as advanced in development, there are several other programs that are targeting the IFN pathway. RSLV-132 is an RNase-Fc fusion protein that enzymatically degrades circulating RNA, thus inhibiting its ability to bind to toll-like receptors (TLR) and activate plasmacytoid dendritic cells. Direct inhibition of TLRs is yet another strategy being employed to target the innate immune system. Immunization with an IFN-alpha-KLH conjugate allows the host to produce his or her own antibodies to IFN-alpha. Plasmacytoid dendritic cells (pDC) are the major producers of type I IFN, and thus they represent a principal target for SLE drug development. BIIB059 is a monoclonal antibody that binds BDCA2, a protein uniquely expressed on pDCs. When BDCA2 is ligated with BIIB059, the protein is internalized, and production of cytokines, chemokines, and interferons is inhibited. In late 2019 , it was announced that two phase 2 studies that evaluated cutaneous lupus as well as SLE achieved their respective end points. Baricitinib, an inhibitor of JAK1 and JAK2, achieved success in a phase 2 SLE study and is currently in phase 3. Let's not forget hydroxychloroquine, which suppresses TLR activation through its inhibition of endosomal acidification.

The future is bright for patients with SLE as research is providing greater insights into SLE pathogenesis that are being translated to drug discovery.

\section{CARDIOVASCULAR DISEASE BURDEN AND BIOMARKERS IN SLE}

Elisabet Svenungsson. Karolinska Institutet, Dept. of Medicine Solna, Karolinska University Hospital, Stockholm, Sweden

\subsection{6/lupus-2020-eurolupus. 10}

Background Premature vascular disease is a major major clinical problem and the major cause of the shortened life expectancy observed among patients with SLE in modern societies. Both ischemic heart disease (IHD) and ischemic cerebrovascular disease (ICVD), is common in SLE, 10\% and 12\% respectively in our cross-sectional Swedish study. The risk estimates for VD in SLE are comparable to diabetes and premenopausal women are at particularly high relative risk. Only a minor part of the vascular risk in SLE is explained by abundance of traditional cardiovascular risk factors Thus, the major reasons for SLE related VD is associated with autoimmunity and SLE per se.

Methods This presentation will review current literature with focus on confirmed and recent findings which can explain why patients with SLE are so commonly affected by VD. Special focus will be given to biomarkers which are associated with the occurrence of VD, and which can serve to select patients in need of preventive treatment.

Results The focus of this presentation will be on SLE related vascular risk factors, but also on vascular outcomes and the temporal relationship between disease onset and VD.

Risk factors seem to differ between various hard vascular outcomes e.g. myocardial infarction, stroke and venous thromboembolism. Many studies use subclinical atherosclerosis or other measures of vascular vulnerability as outcomes, these are important to study but they should be distinctly separated from hard vascular events.

The following patient subgroups/biomarkers will be discussed in the context of SLE related VD: 1) Nephritis and impaired renal function, 2) Antiphospholipid antibodies 3) Complement activation, 4) Systemic inflammation 5) Genetic predisposition. In the clinic there is an interaction between all these factors and also traditional cardiovascular risk factors, which result in high risk for VD. Another approach is to subgroup SLE patients depending on autoantibodies and thus identify subgroups of patients with high risk for VD.

Conclusions Through early mapping and recognition of vascular risk factors in patients with SLE, it should in the future be possible to tailor preventive treatments to individual patients. The goal is to reduce and eventually prevent the heavy burden of vascular disease among patients with SLE. 